\title{
Identification of LCA-binding Glycans as a Novel Biomarker for Esophageal Cancer Metastasis using a Lectin Array-based Strategy
}

Min $\mathrm{Xia}^{1,2^{*}}$, Jun Shao ${ }^{2^{*}}$, Meimei Qiao ${ }^{2^{*}}$, Zhiguo Luo ${ }^{1}$, Xinzhou Deng ${ }^{1}$, Qing Ke ${ }^{1}$, Xiaoxia Dong ${ }^{\circledR}$ and Li Shen ${ }^{1,2,4 \bowtie}$

1. Department of Clinical Oncology, Taihe Hospital, Hubei University of Medicine, Shiyan, Hubei 442000, P.R. China.

2. Department of Biochemistry, School of Basic Medical Sciences, Hubei University of Medicine, Shiyan, Hubei 442000, P.R. China.

3. Department of Pharmacology, School of Basic Medical Sciences, Hubei University of Medicine, Shiyan, Hubei 442000, P.R. China.

4. Hubei Key Laboratory of Embryonic Stem Cell Research, Hubei University of Medicine, Shiyan,Hubei 442000, P.R. China.

*These authors contributed equally to this work.

$\triangle$ Corresponding authors: Li Shen. Department of Clinical Oncology, Taihe Hospital, Hubei University of Medicine, 30 South Renmin Road, Shiyan, Hubei 442000, P.R.China. E-mail: shenlihb@taihehospital.com; Xiaoxia Dong. Department of Pharmacology, School of Basic Medical Sciences, Hubei University of Medicine, 30 South Renmin Road, Shiyan, Hubei 442000, P.R. China, E-mail: xiaoxia.28@163.com.

() The author(s). This is an open access article distributed under the terms of the Creative Commons Attribution License (https://creativecommons.org/licenses/by/4.0/). See http://ivyspring.com/terms for full terms and conditions.

Received: 2020.01.09; Accepted: 2020.05.16; Published: 2020.06.01

\begin{abstract}
Esophageal cancer (EC) is a unique and heterogeneous disease diagnosed mostly at advanced stages. Altered glycans presented on cell surfaces are involved in the occurrence and development of malignancy. However, the effects of glycans on EC progression are largely unexplored. Here, a lectin array was utilized to detect the glycan profiling of the normal esophageal mucosal epithelial cell line and two EC cell lines. The binding of Lens culinaris lectin (LCA) to EC cells was found to be stronger than that of the normal cells. Lectin immunohistochemical staining revealed that LCA-binding glycans were markedly elevated in EC tissues compared to adjacent non-cancerous tissues. LCA staining was significantly associated with lymph node metastasis, depth of invasion, TNM stage and poor overall survival of EC patients. Added LCA to block LCA recognized glycans could inhibit the migration and invasion of EC cells. Further analysis revealed that blocking the biosynthesis of LCA-binding glycans by tunicamycin attenuated cellular migratory and invasive abilities. Additionally, a membrane glycoprotein CD147 was recognized as a binder of LCA. There was a positive correlation between LCA-binding glycans and CD147 expression in clinical samples. Interestingly, CD147 inhibition also reduced cell migration and invasion. These findings indicated that LCA-binding glycans may function as a novel indicator to predict metastasis for patients with EC.
\end{abstract}

Key words: Lectin, glycan, esophageal cancer, metastasis

\section{Introduction}

As one of the most common malignant tumors, esophageal cancer (EC) is a unique and heterogeneous disease diagnosed mostly at advanced stages. This disease ranks seventh in terms of incidence $(572,000$ new cases) and sixth in mortality overall $(509,000$ deaths) in the world [1]. For many years, EC has been a serious public health problem in China [2]. EC has a high incidence of recurrence and metastasis, with a 5 -year overall survival rate of $<20 \%$ [3]. Although significant advances in EC treatment have been achieved by surgery, chemotherapy or radiotherapy, the prognosis for EC patients remains poor. Thus, exploring novel mechanisms and critical signaling pathways will help to the improvement of current therapeutic strategies for EC.

Glycans are highly variable and structurally diverse compounds presented on cell surfaces [4]. The diversity in glycan structures enables them to participate in the occurrence and progression of various tumors [5]. Aberrant glycans can serve as 
prominent biomarkers for cancer detection and act as effective therapeutic targets for anti-cancer drugs $[6,7]$. Recently, increasing studies have found that the invasion and metastasis of various tumors are linked to altered cell-surface glycans. For example, changes in O-glycans were reported to be involved in the invasive behavior of oral squamous cell carcinoma [8]. Artificial modification of cell-surface sialic acid modulated cell invasion in malignant lymphoma [9]. Increased GlcNAc-branched N-glycan levels were closely related to metastasis of glioma, colon cancer, gastric cancer and hepatocellular carcinoma [10-12]. However, the effects of glycans on EC progression are largely unexplored. In our previous study, we confirmed that inhibition of core 1 O-glycans attenuated irradiation-enhanced migration and invasion of EC cells [13]. We, therefore, wanted to gain further insight into the mechanisms underlying glycans-mediated metastasis in EC.

Lectins have been the principal means of investigating cell surface glycan patterns [14]. The lectin microarray is an emerging and straightforward technology for glycomic analysis [15]. In the present study, we explored the differences in glycans between the normal esophageal mucosal epithelial cell line and two EC cell lines using a lectin microarray. Our data demonstrated for the first time that Lens culinaris lectin (LCA) specifically bound to metastasisassociated glycans in EC.

\section{Materials and Methods}

\section{Cell lines and culture}

Human EC cell lines (Eca-109 and KYSE-150), obtained from Procell (Wuhan, China), were maintained in DMEM (Gibco-BRL, Carlsbad, CA, USA) supplemented with $10 \%$ fetal bovine serum (FBS; HyClone, Chicago, IL, USA). Human normal esophageal mucosal epithelial cell line Het-1A, obtained from ATCC (Manassas, VA, USA), was maintained in BEGM medium kit (Lonza/Clonetics Corporation, Walkersville, MD, USA). All cells were cultured in a $5 \% \mathrm{CO}_{2}$ atmosphere at $37^{\circ} \mathrm{C}$.

\section{Lectin microarray analysis}

Lectin microarray (purchased from BC Biotechnology, Guangdong, China) was produced using 37 lectins with different glycan-binding specificities [16]. Lectin microarray screening for cell surface glycans was performed in our lab as described previously [17]. Briefly, the harvested Eca-109, KYSE150, and Het-1A cells were washed 3 times with cold phosphate-buffered saline (PBS), marked with CFDASE (Life Technologies, Carlsbad, CA, USA), and probed on a lectin microarray [17]. The signal intensities of greater than or equal to 3 standard deviations (SD) above background, which scanned by a GenePix 4200B scanner (Molecular Devices, Sunnyvale, CA, USA), were considered as positive signals $[16,18]$.

\section{Tissue samples and immunohistochemical (IHC) staining}

A total of $91 \mathrm{EC}$ and 31 adjacent non-tumor tissues were acquired from patients who had undergone surgical treatment in the Taihe Hospital, Hubei University of Medicine (Hubei, China). Inclusion criteria: (1) patients were pathologically diagnosed with esophageal cancer; (2) patients had not received chemical treatment or physical therapy before surgery; (3) patients with complete clinical data. Exclusion criteria: (1) patients with other malignant tumors; (2) patients without complete clinical data. All patients consented to the use of their tissue samples. This study was approved by the Research Ethics Committee of the Hubei University of Medicine. IHC staining was carried out following a standard protocol [10]. To detect the expression of LCA-binding glycans, LCA (L-1040, Vector Labs, Burlingame, CA, USA) served as the primary antibody. Horseradish peroxidase (HRP)-conjugated streptavidin (Beyotime, Jiangsu, China) was used as the secondary antibody (Beyotime). To measure CD147 expression, the samples were incubated with the primary antibody (anti-CD147, ab64616, Abcam, Cambridge, MA, USA) and detected with an HRPlabeled secondary antibody. The extent and intensity of IHC staining were measured as previously described [18]. A staining index (with values from 0 to 12) was obtained as the staining intensity (negative $=0$, weak $=1$, moderate $=2$, or strong $=3$ ) multiplied by the proportion of immunopositive tumor cells $(0-25 \%=1$, $25-50 \%=2,50-75 \%=3$, or $75-100 \%=4$ ).

\section{Transwell migration and invasion assays}

Using a serum-free medium, the density of Eca109 and KYSE-150 was adjusted to $2 \times 10^{5}$ cells $/ \mathrm{ml}$. Then $200 \mu$ l of cell suspension was added to the upper chamber of an insert $(8.0 \mu \mathrm{m}$ pore size; Costar, Cambridge, MA, USA). The lower chamber was filled with medium containing 20\% FBS [19]. To monitor cell invasion, the upper chambers of transwell inserts were coated with matrigel. To monitor cell migration, no matrigel was coated in the upper chambers. After $24 \mathrm{~h}$ of incubation, cells on the underside were stained with $0.1 \%$ crystal violet (Beyotime). Images were taken at $200 \times$ magnification.

\section{Cell viability assay}

Eca-109 and KYSE-150 cells $\left(5 \times 10^{3}\right.$ cells/well) were seeded into 96-well plates. One day later, cells from triplicate wells were treated with the indicated 
different concentrations of LCA (Vector Labs), tunicamycin (Sigma, St. Louis, MO, USA), or CD147 blocking antibody (Fitzgerald, MA, USA). Following incubation for $24 \mathrm{~h}$, cell viability in each group was determined using the Cell Counting Kit-8 (CCK-8; Beyotime). Absorbance at $450 \mathrm{~nm}$ was measured using a microplate reader (BioTek, Germany). The $50 \%$ inhibitory concentration (IC50) was calculated through the formula [20].

\section{Lectin blotting and western blotting analysis}

RIPA lysis buffer (Beyotime) was used to extract total protein. A BCA Protein Quantitative Kit (Beyotime) was adopted to detect protein concentrations. Protein was separated by SDS-PAGE (10\% gel) and transferred to PVDF membranes (Millipore, Billerica, MA, USA). For lectin blotting, the membranes were blocked with a carbo-free blocking solution (Vector Labs) and subsequently incubated with biotin-labeled LCA. For western blotting, the membranes were blocked with 5\% non-fat milk. Primary antibodies (Absin, Shanghai, China) were used including anti-MMP2, anti-MMP-9, anti-p53, anti-Bcl-xL, anti-CylinD1, anti-BAX and anti-pAkt. The signal was evaluated using an enhanced chemiluminescence detection kit (Beyotime). An antibody against GAPDH (Absin) served as an endogenous reference.

\section{Lectin pull-down and LC-MS/MS analysis}

To capture the glycoproteins, proteins from whole-cell extractions were incubated with LCAconjugated agarose beads (Vector Labs) at $4^{\circ} \mathrm{C}$ overnight. After washing three times with PBS, samples were separated by SDS-PAGE on a $10 \%$ gel and stained with coomassie blue R250 (Beyotime).
In-gel trypsin digestion and LC-MS/MS analysis were done as previously reported [21].

\section{Statistical analysis}

All the values obtained from at least three independent experiments were analyzed using GraphPad Prism 5.0 (La Jolla, CA, USA). Data were expressed as mean \pm SD. Comparisons were conducted by student's t-test or one-way analysis of variance (ANOVA). Kaplan-Meier method was applied to determine overall survival rates. $\mathrm{P}<0.05$ was considered to be significant.

\section{Results}

\section{Identification of metastasis-associated glycans on EC cell lines}

To identify which glycans appear and how cancer cells differ from normal cells, Het-1A, Eca-109, KYSE-150 cell lines were chosen for this study. The differences in glycans were compared by a lectin microarray, in which each lectin was present in triplicate (Fig. 1A). The specific glycans recognized by the lectins and the relative fold change of each lectin were summarized in Table 1 . We found that lectins Jacalin, LCA, AAL, PHA-L, and UEA-I exhibited stronger binding affinities to cancer cells, as compared to normal cells (Fig. 1B). The most significant difference was seen for LCA. It has been reported that cancer cells frequently possess higher metastatic properties than normal cells [22,23]. Based on this information, we speculated that LCA-binding glycans played an important role in EC metastasis. We, therefore, selected it for further analysis.
A

\begin{tabular}{|c|c|c|c|}
\hline$B \subseteq A$ & Jacalin & ECA & HiHA \\
\hline WFA & GSSL-H & M,AL.-HI & PHIA-E \\
\hline$A \wedge . L$ & UEEA-i & $P N A$ & EFE! \\
\hline PTI_..' & I_TL & MFL & LELL. \\
\hline GSL-! & $\mathrm{DE}, \mathrm{A}$ & $D S A$ & RCA1?20 \\
\hline STL & BS. & BFL & ConA \\
\hline PTi_-i! & ICA & SESA & VIA \\
\hline NPA & $P S A$ & $\hat{A C A}$ & NGAA \\
\hline$S i A$ & PVị! & MíAi-i & GN,A. \\
\hline PHA I & ESA & SNA & Matker \\
\hline
\end{tabular}

B Eca-109
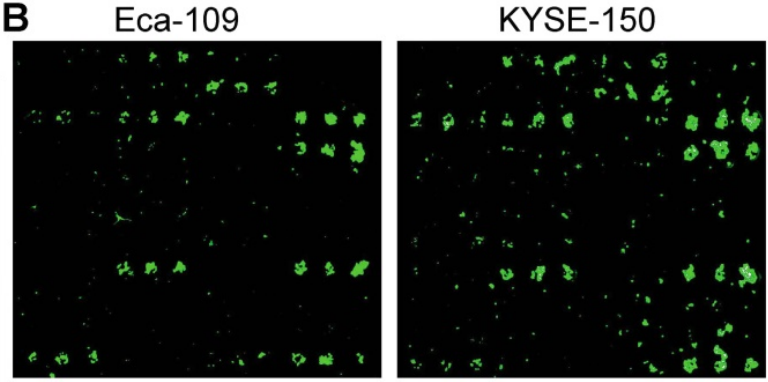

Het-1A

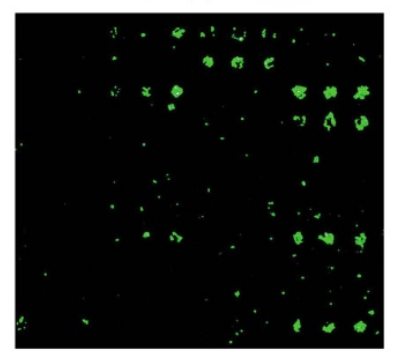

Figure 1. Identification of metastasis-specific lectin binding to EC cells. (A) Layout of the lectin microarray containing 37 lectins. (B) Representative binding patterns of Het-1A, Eca-109 and KYSE-150 cell lines. 
Table 1. The relative fold change of each lectin in Eca-109 and KYSE-150 cells

\begin{tabular}{|c|c|c|c|c|}
\hline \multirow[t]{2}{*}{ Lectin } & \multirow[t]{2}{*}{ Origin } & \multirow[t]{2}{*}{ Preferred glycan structures } & \multicolumn{2}{|c|}{ Compared with Het-1A (Fold change) } \\
\hline & & & Eca-109/Het-1A & KYSE-150/Het-1A \\
\hline Jacalin & Artocarpus integrifolia & Galß1-3GalNAca-Ser/Thr(T) and GalNACa-Ser/Thr(Tn) & $1.88^{*}$ & $2.54^{*}$ \\
\hline ECA & Erythrina cristagalli & Gal $\beta-1,4$ GlcNAc & 0.74 & 1.34 \\
\hline HHL & Hippeastrum hybrid & Polymannose $(\alpha-1,3)$ and $(\alpha-1,6)$ linked mannose & - & - \\
\hline WFA & Wisteria floribunda & GalNAca $/ \beta-1,3 / 6 \mathrm{Gal}$ & - & - \\
\hline GSL-II & Griffonia simplicifolia & GlcNAc & - & - \\
\hline MAL-II & Maackia amurensis & Sia2-3Galß1-4Glc (NAc) & 1.14 & 1.09 \\
\hline PHA-E & Phaseolus vulgaris & Bisecting GlcNAc and biantennary N-glycans & - & - \\
\hline PTL-I & Psophocarpus tetragonolobus & aGalNAc & - & - \\
\hline SJA & Sophora japonica & Terminal in GalNAc and Gal & - & - \\
\hline PNA & Arachis hypogaea & Galß1-3GalNAca-Ser/Thr(T) & - & - \\
\hline EEL & Euonymus europaeus & Gala1-3(Fuca1-2)Gal & 1.03 & 1.17 \\
\hline AAL & Aleuria aurantia & Fuca-1,6GlcNAc, Fuca-1,3LacNAc & $1.97^{*}$ & $2.41^{*}$ \\
\hline LTL & Lotus tetragonolobus & a-L-Fuc & - & - \\
\hline MPL & Maclura pomifera & aGalNAc & - & - \\
\hline LEL & Lycopersicon esculentum & Poly-LacNAc and (GlcNAc)n & 1.29 & 1.36 \\
\hline GSL-I & Griffonia simplicifolia & aGalNAc, aGal & - & - \\
\hline DBA & Dolichos biflorus & GalNAca-Ser/Thr(Tn) & - & - \\
\hline LCA & Lens culinaris & a-Man and Fuca-1,6GlcNAc (core fucose) & $2.75^{*}$ & $3.44^{*}$ \\
\hline RCA120 & Ricinus communis & Gal and GalNAc & - & - \\
\hline STL & Solanum tuberosum & (GlcNAc)n & - & - \\
\hline BS-I & Bandeiraea simplicifolia & aGal and aGalNAc & - & - \\
\hline ConA & Canavalia ensiformis & a-Man (inhibited by presence of bisecting GlcNAc) & - & - \\
\hline PTL-II & Psophocarpus tetragonolobus & Gal & - & - \\
\hline DSA & Datura stramonium & $\beta 1-4 G l c N A c$ and LacNAc & - & - \\
\hline SBA & Glycine max & Terminal GalNAc (especially GalNAca1-3Gal) & - & - \\
\hline VVA & Vicia villosa & GalNAc and GalNAca-Ser/Thr(Tn) & 1.37 & 1.49 \\
\hline NPA & Narcissus pseudonarcissus & Polymannose(a-1,6)linked mannose & - & - \\
\hline PSA & Pisum sativum & Fuca-N-acetylchitobiose -man & - & - \\
\hline ACA & Amaranthus caudatus & Galß1-3GalNAca-Ser/Thr(Tn) & - & - \\
\hline WGA & Triticum unlgaris & Terminal GlcNAc and (GlcNAc)n & - & - \\
\hline UEA-I & Ulex europaeus & Fucosea1-2Galß1-4Glc(NAc) & $1.74^{*}$ & $2.33^{*}$ \\
\hline PWM & Phytolacca americana & GlcNAc & - & - \\
\hline MAL-I & Maackia amurensis & Gal $\beta-1,4 \mathrm{GlcNAc}$ and Sia2-3Gal $\beta 1-4 \mathrm{Glc}(\mathrm{NAc})$ & - & - \\
\hline GNA & Galanthus nivalis & $(a-1,3)$ Man & - & - \\
\hline $\mathrm{BPL}$ & Bauhinia purpurea alba & Galß1-3GalNAc & - & - \\
\hline PHA-L & Phaseolus vulgaris & Tri/Tetra-antennary complex-type N-glycan & $2.61^{*}$ & $2.21^{*}$ \\
\hline SNA & Sambucus nigra & Sia2-6Gal $\beta 1-4 G l c(N A c)$ & 1.01 & - \\
\hline
\end{tabular}

Signal intensities obtained from lectin microarrays were normalized. ${ }^{*} \mathrm{P}<0.05$; -: Negative signal.

\section{Validation of metastasis-specific LCA-binding patterns in EC patients}

LCA staining was found to be significantly elevated in EC tissues as compared to adjacent nontumor tissues $(\mathrm{P}<0.05$; Fig. 2A-B). A significant association between the positive staining of LCA and the occurrence of lymph node metastasis, depth of invasion, and TNM stage was observed in patients (Table 2). However, no significant differences were detected between the binding tendency of LCA and gender, age, tumor size, or differentiation. Among EC tissues, LCA staining intensities in the metastasispositive group were markedly higher than those in the metastasis-negative group $(\mathrm{P}<0.05$; Fig. $2 \mathrm{C})$. Kaplan-Meier survival analysis illustrated that, compared with those showing low LCA staining; patients with high LCA staining had shorter overall survival $(\mathrm{P}<0.05 ;$ Fig.2D). Our results strongly suggested that the presence of LCA-specific glycans could be used as a useful biomarker of metastatic potential in EC.

\section{Added LCA to block LCA-binding glycans inhibits migration and invasion of EC cells}

To explore whether lectin LCA affected any processes associated with metastasis, migration and invasion were studied by transwell assays. We observed that the migration of Eca-109 and KYSE-150 cells was suppressed by LCA in a dose-dependent manner, particularly at a higher concentration (Fig. 3A). Compared with the control group, LCA dose-dependently reduced the number of invaded Eca-109 and KYSE-150 cells (Fig. 3B).

To investigate the mechanisms underlying the anti-metastatic effects of LCA, cells were firstly incubated with different concentrations of LCA. As shown in Fig. 3C, cell viability decreased linearly with 
increasing LCA concentrations. Correspondingly, the IC50 values of Eca-109 and KYSE-150 were $6 \mu \mathrm{g} / \mathrm{ml}$ and $7 \mu \mathrm{g} / \mathrm{ml}$. Then cells were treated with LCA at the IC50 concentration. It was reported that the matrix metalloproteinases (MMPs) and PI3K/Akt signaling pathway-related proteins (p53, CylinD1, Bcl-2, Bax, and pAkt) regulated the invasion and metastasis in many types of cancer cells [24-26]. Thus, the expression of related molecules was analyzed by western blotting. We found that the levels of MMP-2, MMP-9, p53, Bcl-xL, CylinD1, BAX, and pAkt were not changed after LCA treatment in Eca-109 and KYSE-150 cells (Fig. 3D). Taken together, these findings provided evidence that added LCA to block LCA-binding glycans inhibited cell migration and invasion. These effects of LCA may not be related to the expression of MMPs and the PI3K/Akt pathway.

\section{Blocking the synthesis of LCA-binding glycans by tunicamycin attenuates the migratory and invasive abilities of EC cells}

Tunicamycin is known to be an inhibitor of the synthesis of N-linked glycans [27]. Results of CCK-8 assay showed that tunicamycin induced anti-proliferative activity, displaying an $\mathrm{IC}_{50}$ of 1 $\mu \mathrm{g} / \mathrm{ml}$ in both cell lines (Fig. 4A). We also found that tunicamycin at the IC50 concentration decreased the migration and invasion of Eca-109 and KYSE-150 cells
(Fig. 4B-C). Besides, lectin blotting analysis confirmed that tunicamycin caused a suppressive effect on the expression of LCA-binding glycans (Fig. 4D). However, tunicamycin had no obvious effects on the levels of MMP-2, MMP-9, p53, Bcl-xL, CylinD1, BAX and pAkt (Fig. 4E). Our results further confirmed a modulating influence of LCA-binding glycans on cell metastasis.

\section{Identification of CD147 as an LCA-recognized membrane glycoprotein}

To identify potential glycoproteins recognized by LCA, lectin pull-down assay was performed (Fig. 5A). As a result, a membrane glycoprotein CD147 was detected both in Eca-109 and KYSE-150 cells (Table S1). LCA immuno-precipitation revealed that CD147 was efficiently co-precipitated with LCA (Fig. 5B). Then we carried out IHC staining and measured CD147 expression in specimens. The results showed that the expression of CD147 in EC tissues was higher than that in adjacent non-tumor tissues $(\mathrm{P}<0.05$; Fig. 5C-D). CD147 expression was considerably correlated with lymph node metastasis, depth of invasion, and TNM stage ( $\mathrm{P}<0.05 ;$ Table 2). Based on each paired IHC score, there was a positive correlation between LCA-binding glycans and CD147 (P < 0.001; Fig. 5E).
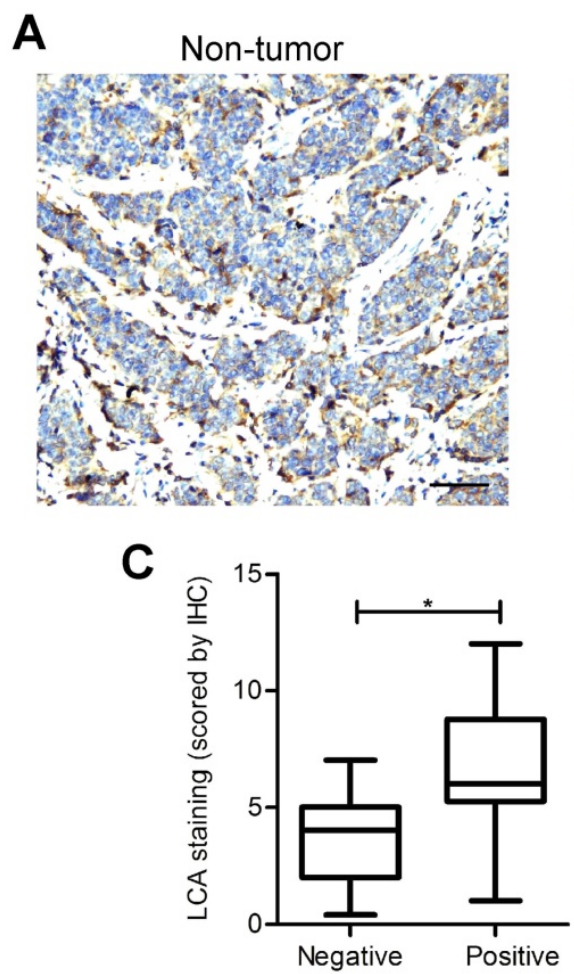

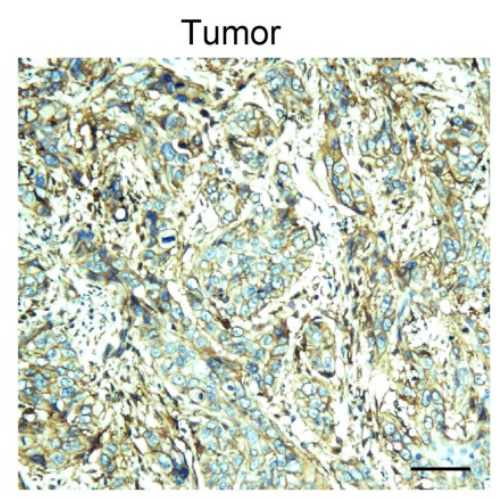

B
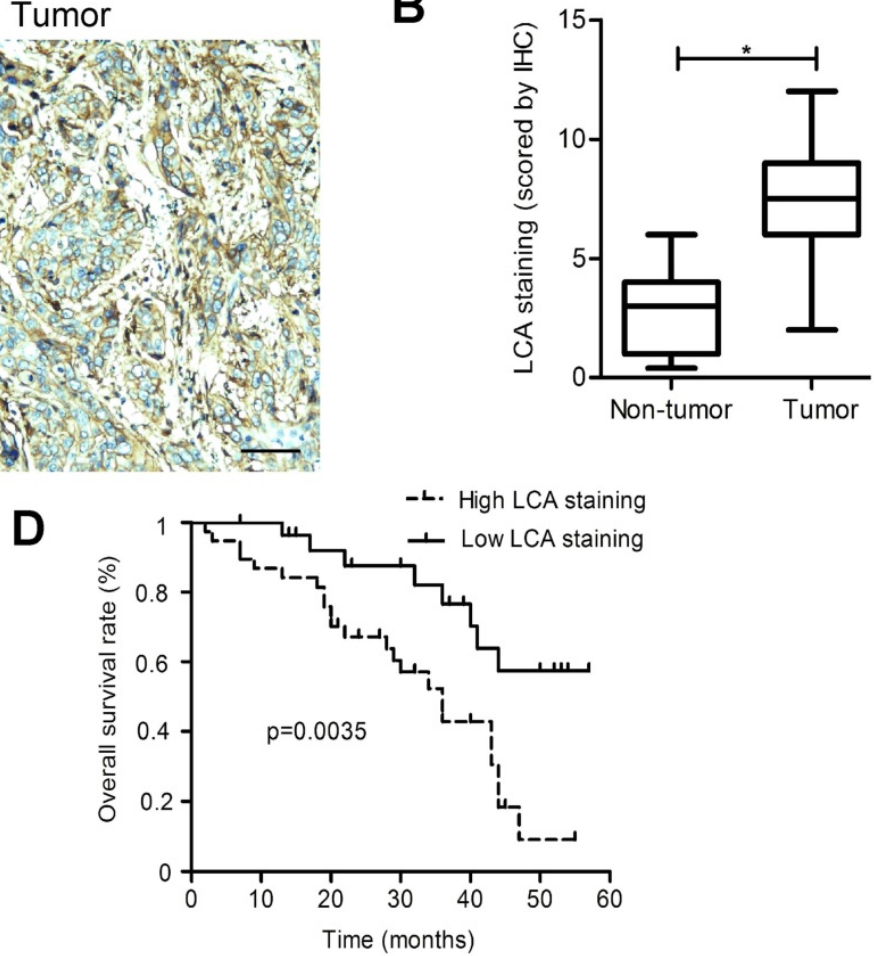

Figure 2. IHC staining for LCA-binding glycans. (A) Representative photomicrographs for LCA-binding glycans in EC and adjacent non-tumor tissues. (B) Analysis of LCA-binding glycans based on the scoring data. (C) Comparison of LCA staining intensities in the metastasis-positive group and metastasis-negative group. (D) Kaplan-Meier survival curves for LCA staining. Scale bar represents $100 \mu \mathrm{m}$. $* \mathrm{P}<0.05$. 
A
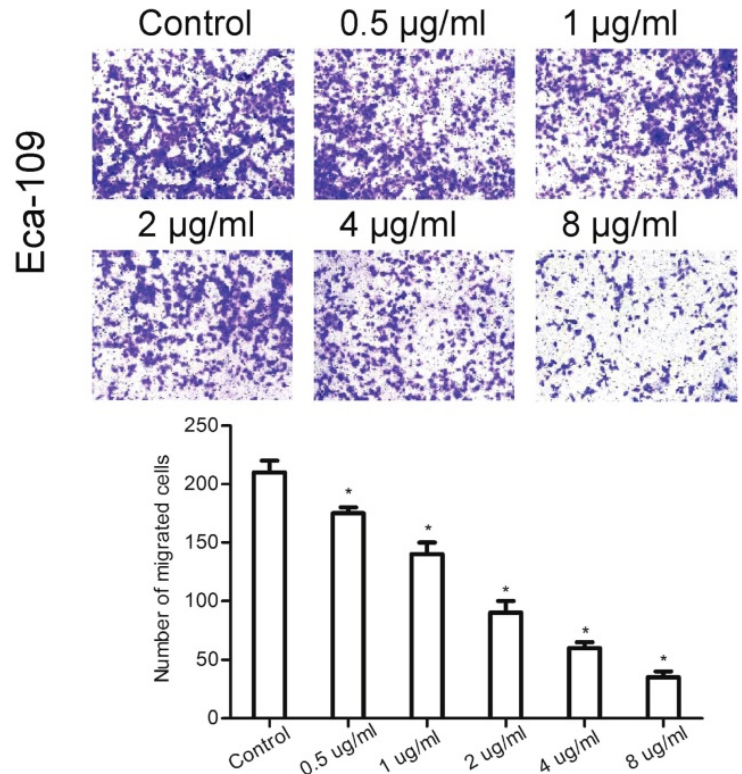

B
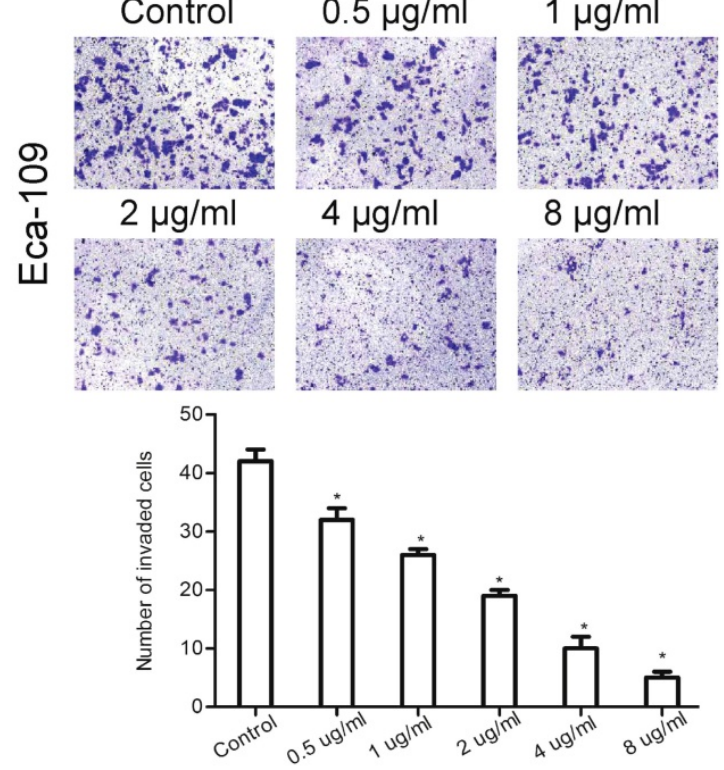

C

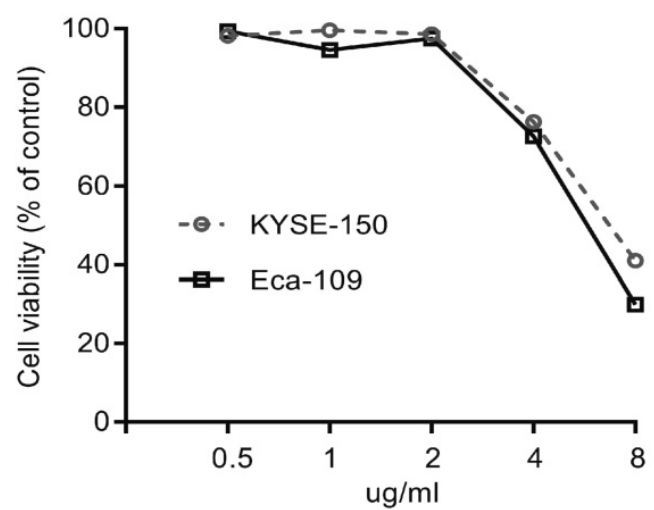

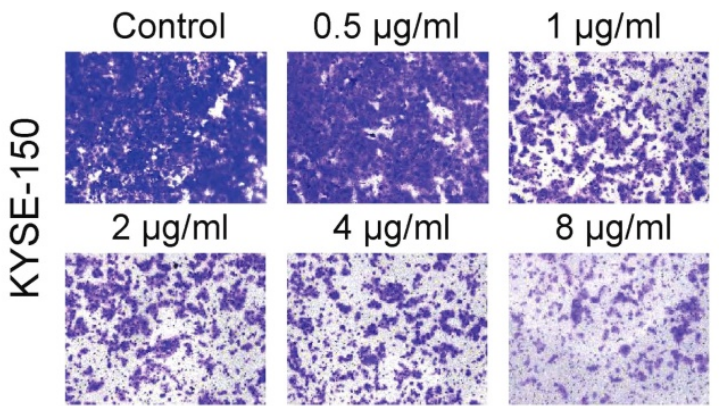

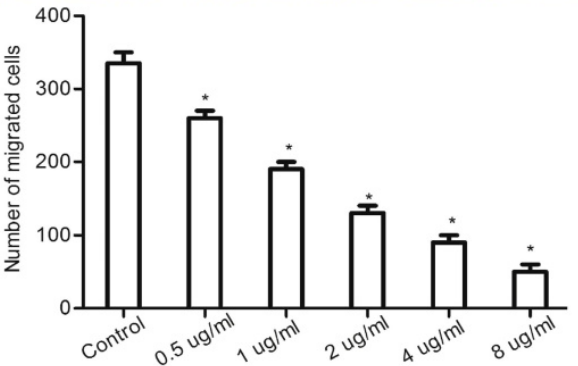

Contro
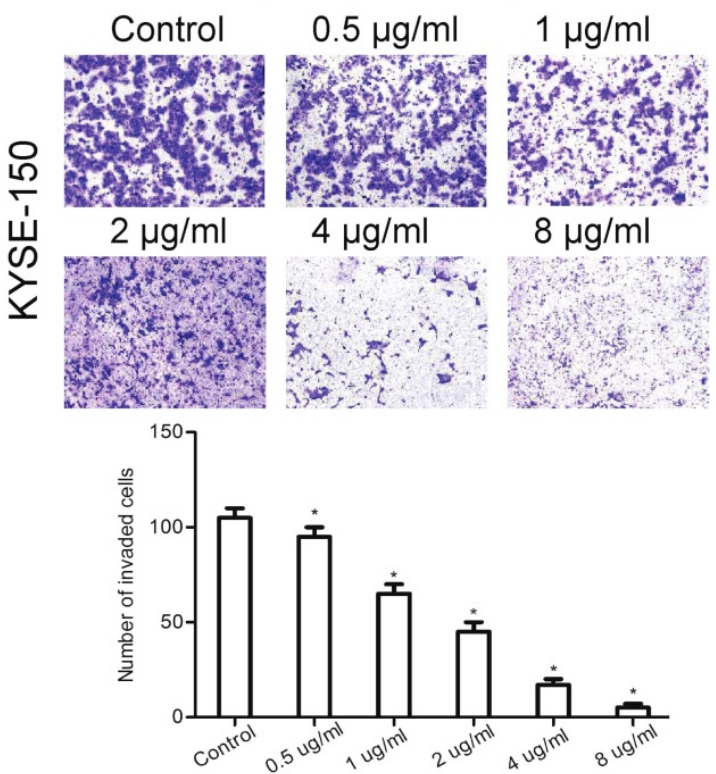

Control LCA Control LCA

D MMP-2
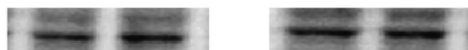

MMP-9

p53

$\mathrm{BCl}-\mathrm{xL}$

CylinD1

BAX

pAkt
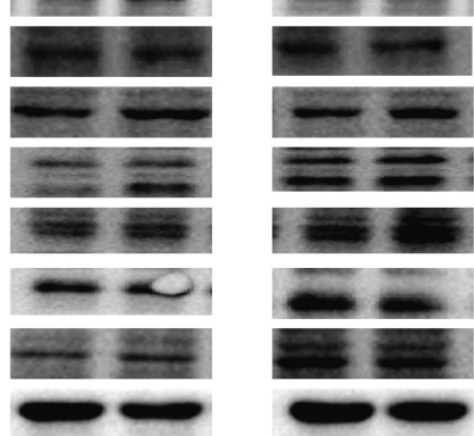

GADPH

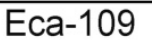

KYSE-150

Figure 3. Effects of LCA on EC cell migration and invasion. (A) Representative images and the mean migration number for each treatment group. (B) Representative images and the mean invasion number for each treatment group. (C) Cell viability for each treatment group. (D) Expression levels of the MMPs and PI3K/Akt pathway-associated proteins for cells treated with LCA at the IC50 concentration. Untreated cells served as a control. * $\mathrm{P}<0.05$ compared to the control group. 
Table 2. Clinicopathological features of esophageal cancer patients

\begin{tabular}{|c|c|c|c|c|c|c|c|}
\hline \multirow[t]{2}{*}{ Variables } & \multirow[t]{2}{*}{ Number } & \multicolumn{2}{|c|}{ LCA staining } & \multirow[t]{2}{*}{$P$-value } & \multicolumn{2}{|c|}{ CD147 expression } & \multirow[t]{2}{*}{$P$-value } \\
\hline & & Low $(n=39)$ & High $(n=52)$ & & Low $(n=31)$ & High $(n=60)$ & \\
\hline \multicolumn{8}{|l|}{ Age } \\
\hline$<60$ & 40 & 20 & 20 & 0.399 & 21 & 19 & 0.603 \\
\hline$\geq 60$ & 51 & 19 & 32 & & 10 & 41 & \\
\hline \multicolumn{8}{|l|}{ Gender } \\
\hline male & 43 & 15 & 28 & 0.621 & 22 & 21 & 0.317 \\
\hline female & 48 & 24 & 24 & & 9 & 39 & \\
\hline \multicolumn{8}{|l|}{ Size of tumor } \\
\hline$<5 \mathrm{~cm}$ & 44 & 22 & 22 & 0.134 & 13 & 31 & 0.281 \\
\hline$\geq 5 \mathrm{~cm}$ & 47 & 17 & 30 & & 18 & 29 & \\
\hline \multicolumn{8}{|l|}{ Differentiation } \\
\hline Moderate-High & 50 & 25 & 25 & 0.129 & 10 & 40 & 0.275 \\
\hline Low & 41 & 14 & 27 & & 21 & 20 & \\
\hline \multicolumn{8}{|c|}{ Depth of invasion } \\
\hline $\mathrm{T} 1+\mathrm{T} 2$ & 36 & 29 & 7 & $0.004^{*}$ & 20 & 16 & $0.013^{*}$ \\
\hline $\mathrm{T} 3+\mathrm{T} 4$ & 55 & 10 & 45 & & 11 & 44 & \\
\hline \multicolumn{8}{|c|}{ Lymph node metastasis } \\
\hline Positive & 61 & 14 & 47 & $0.001^{*}$ & 11 & 50 & $0.002^{*}$ \\
\hline Negative & 30 & 25 & 5 & & 20 & 10 & \\
\hline \multicolumn{8}{|l|}{ TNM stage } \\
\hline $\mathrm{I}+\mathrm{II}$ & 45 & 27 & 18 & $0.015^{*}$ & 24 & 21 & $0.016^{*}$ \\
\hline III +IV & 46 & 12 & 34 & & 7 & 39 & \\
\hline
\end{tabular}
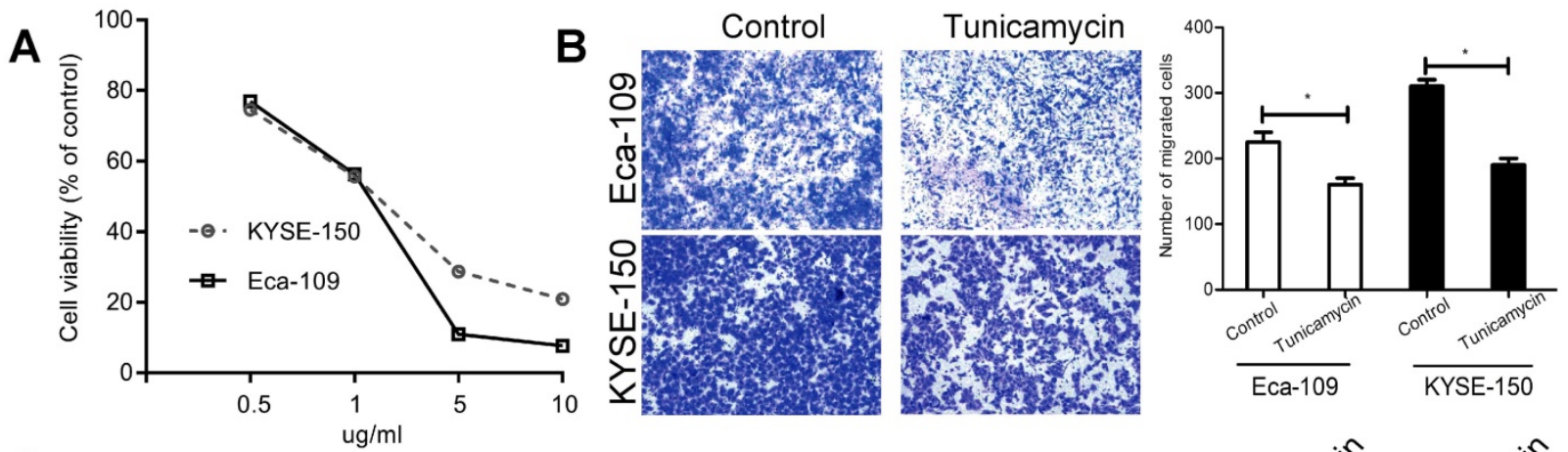

C

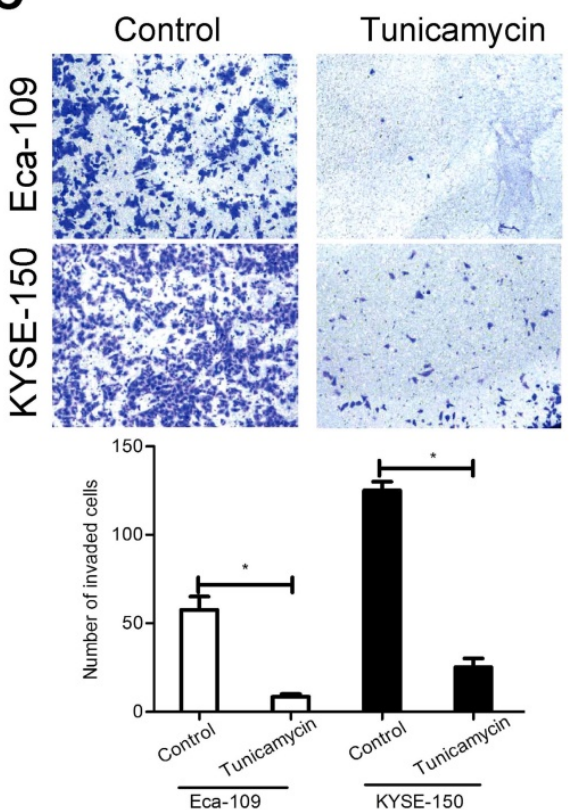

D

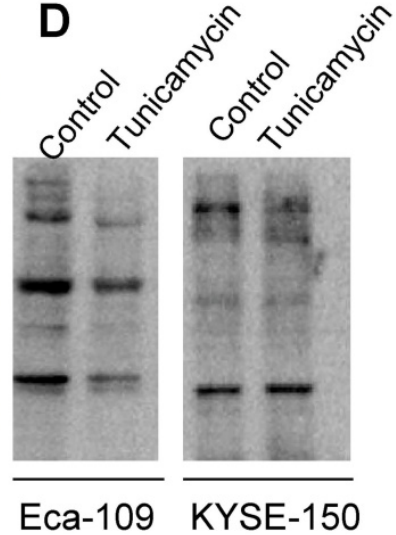

E

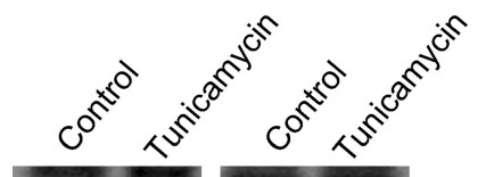

MMP-2

MMP-9

p53

$\mathrm{BCl}-\mathrm{xL}$
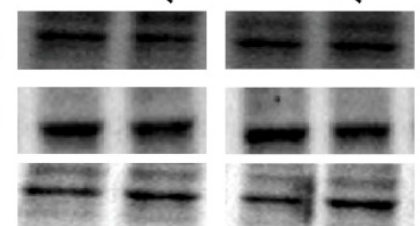

CylinD1

BAX
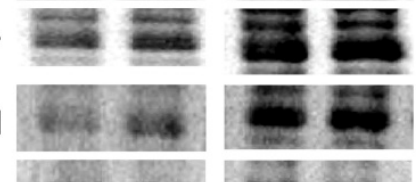

pAkt

GADPH

Eca-109
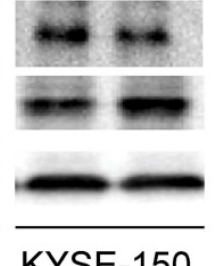

Figure 4. Effects of tunicamycin on EC cell migration and invasion. (A) Cell viability for each treatment group. (B) Representative images and the mean migration number for cells treated with tunicamycin at the IC50 concentration. (C) Representative images and the mean invasion number for cells treated with tunicamycin at the IC50 concentration. (D) Expression levels of LCA-binding glycans for cells treated with tunicamycin at the IC50 concentration. (E) Expression levels of the MMPs and PI3K/Akt pathway-associated proteins for cells treated with tunicamycin at the IC50 concentration. Cells treated with DMSO served as a control. * P $<0.05$ compared to the control group. 


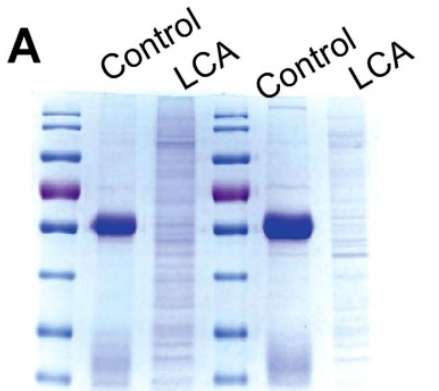

Eca-109 KYSE-150

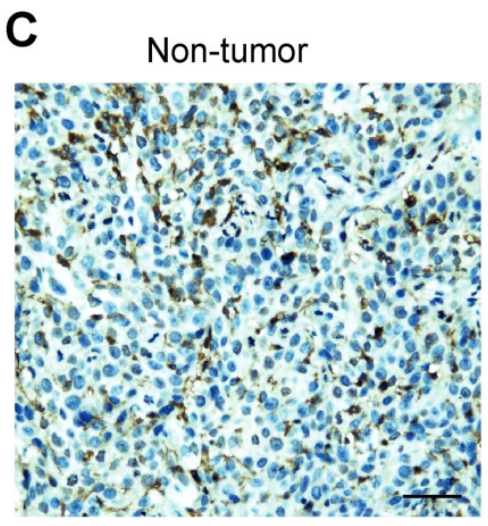

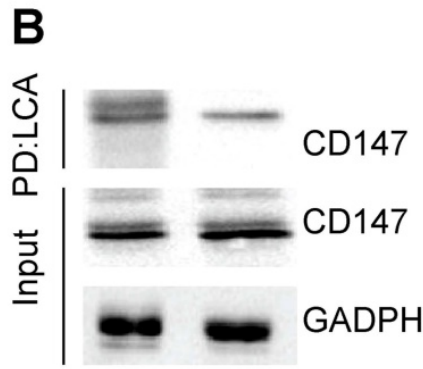

Eca-109 KYSE-150
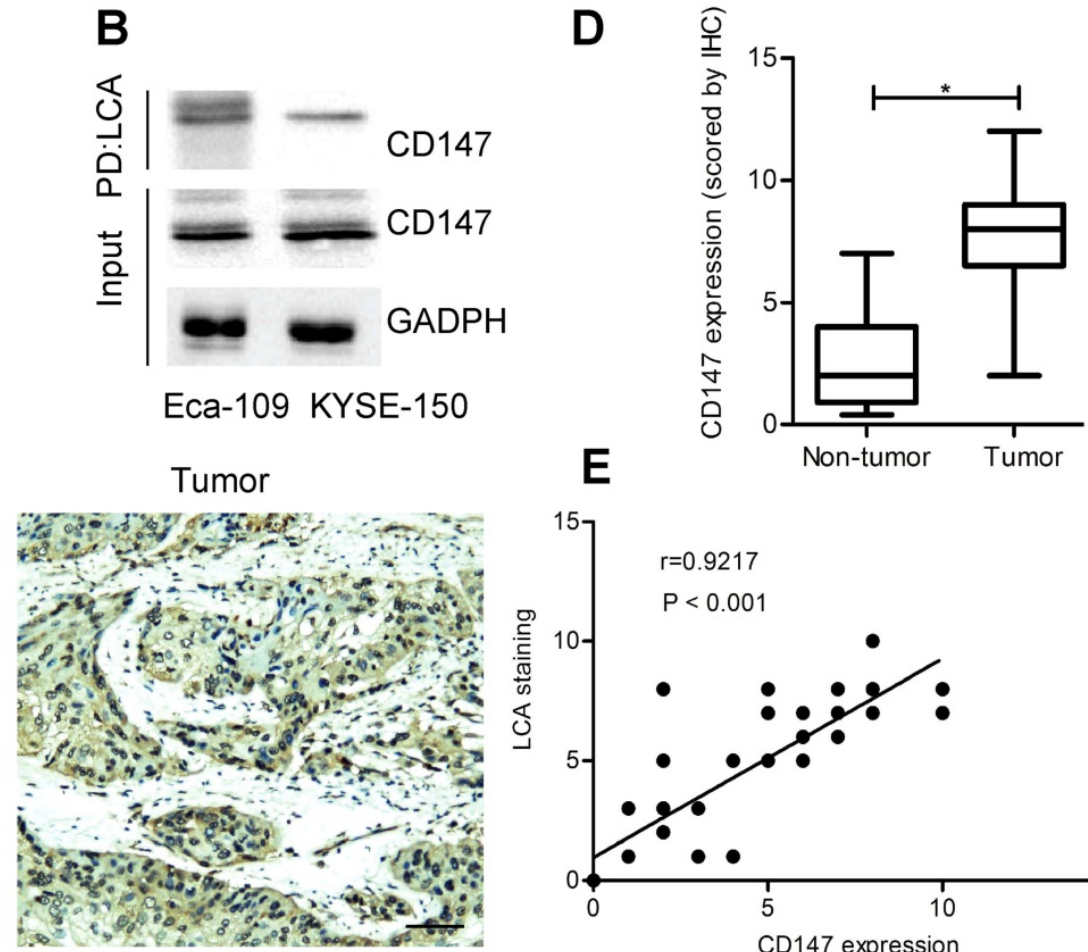

$\mathrm{E}$

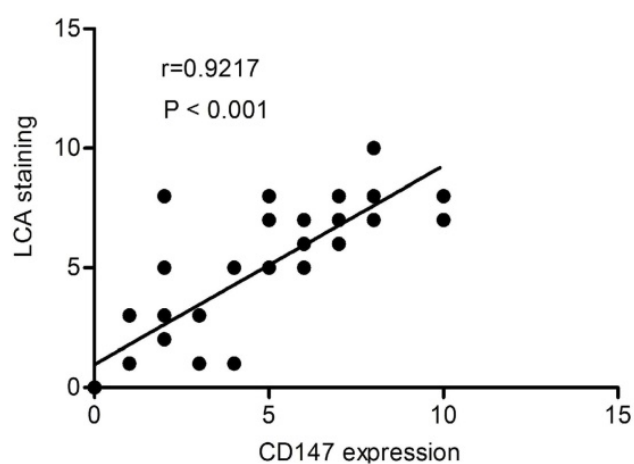

Figure 5. Identification of CD147 as an LCA-recognized glycoprotein. (A) Coomassie blue staining for immunoprecipitated samples. (B) LCA immunoprecipitation assay for Eca-109 and KYSE-150 cells. PD: pull down. (C) Representative photomicrographs for CD147 expression in EC and adjacent non-tumor tissues. Scale bar represents $100 \mu \mathrm{m}$. (D) Analysis of CD147 expression based on the scoring data for IHC (E) Correlation between LCA-binding glycans and CDI47 expression in EC tissues. IgG served as a control. $\mathrm{P}<0.05$

\section{Inhibition of CD147 reduces cell migration and invasion}

As described above, LCA-binding glycans had a strong impact on cell migration and invasion. To determine whether these effects were mediated by the LCA-CD147 interaction, EC cells were cultured in the presence of CD147 function-blocking antibody at a lower concentration. We found that the antibody treatment decreased the migration ability and the invasiveness of Eca-109 and KYSE-150 cells (Fig. 6A-B). These data suggested that LCA-binding glycans regulated cell metastasis, at least in part, via modulation of CD147.

\section{Discussion}

Metastasis is a major contributor to the mortality of cancer patients [28]. This process is associated with cancer cell extravasation and subsequent invasion of normal surrounding tissue, which is mediated by cell-surface mechanisms [16]. Cell surface-associated glycans regulate many cellular processes, including adhesion, migration, signaling, and extracellular matrix organization [29]. Growing evidence has shown that aberrantly expressed glycans recognized by lectins can serve as important regulators for cancer metastasis $[8,12,18,30]$. In the current study, we demonstrated for the first time that lectin LCA specifically bound to metastasis-associated cellsurface glycans in EC. The expression of LCA-binding glycans was positively correlated with lymph node metastasis, depth of invasion, TNM stage and poor overall survival of patients. We also confirmed that inhibition of LCA-binding glycans could suppress EC cell migration and invasion. Our findings provided a novel insight into the mechanisms responsible for EC metastasis.

Several molecules and pathways have been proven to contribute to EC cell migration and invasion. For example, amplified or overexpressed MMP-2 and MMP-9 were associated with the invasive and metastatic properties of EC cells [31,32]. The PI3K/Akt pathway was an important signaling pathway that modulated cell migration and invasion in EC $[7,33]$. However, whether LCA-binding glycans participate in EC metastasis mediated by the MMPs and PI3K/Akt pathway is still unknown. In this study, after examining the inhibition effects of LCA and tunicamycin on migration and invasion, we then investigated the levels of MMPs and the PI3K/Akt signaling pathway-related proteins. Western blotting showed that LCA and tunicamycin had no obvious effects on the levels of MMP-2, MMP-9, p53, Bcl-xL, CylinD1, BAX and pAkt. Therefore, blocking the biosynthesis of LCA-binding glycans inhibited EC cell 
migration and invasion maybe not via the MMPs and PI3K/Akt signaling pathway.

A
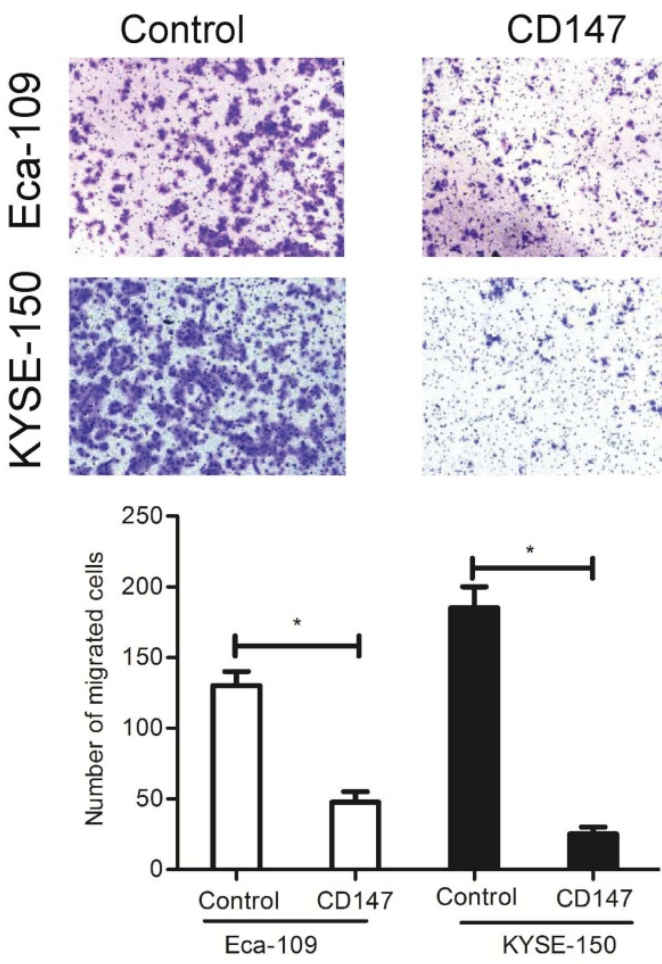

B

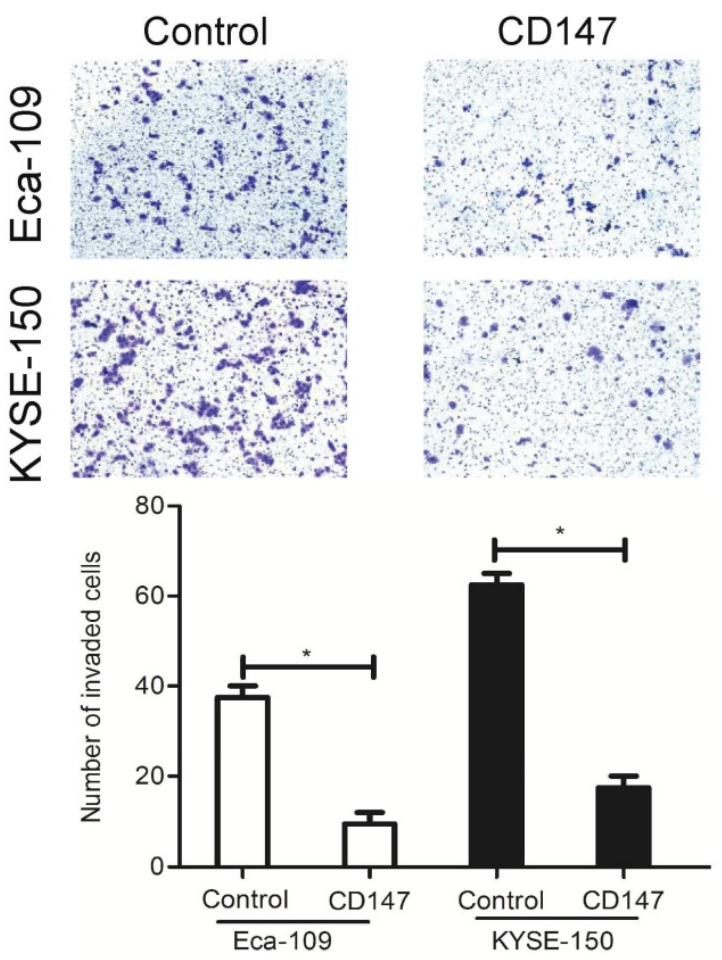

Figure 6. Effects of CD147 on EC cell migration and invasion. Eca-109 and KYSE- 150 cells were pre-treated with $1 \mu \mathrm{g} / \mathrm{ml}$ and $2 \mu \mathrm{g} / \mathrm{ml} \mathrm{CD147} \mathrm{blocking} \mathrm{antibody,}$ respectively. (A) Representative images and the mean migration number for each group. (B) Representative images and the mean invasion number for each group. IgG served as a control. $* \mathrm{P}<0.05$ compared to the control group.

CD147, also known as EMMPRIN, Basign, and HAb18G, is a widely expressed cell surface protein with multiple glycosylated forms. Previous studies have shown that lectins PHA-L, PHA-E, and LEL could directly bind to CD147 in many types of cancer cells $[12,34,35]$. In the present study, we identified CD147 as a lectin LCA-recognized membrane glycoprotein in EC cells. Recently, the functional roles of CD147 in cancer proliferation, migration, and invasion have been extensively reported. For instance, inhibition of CD147 expression decreased proliferation and invasion in colorectal cancer stem cells [36]. In vitro and in vivo prostate cancer metastasis can be modulated by CD147 [37]. CD147 was a key regulator of metastasis in non-small cell lung cancer [19], bladder cancer [38], EC [39] and gastric cancer [40]. Our findings agree with these reports that CD147 was upregulated in EC tissues. Moreover, the antibody blockade of CD147 reduced cell migration and invasion. Thus, preventing the binding of CD147 to LCA-specific glycans by CD147 blocking antibody was also involved in EC metastasis.

In summary, lectin LCA specifically bound to metastasis-associated cell-surface glycans in EC. Additionally, a membrane glycoprotein CD147 was recognized as a binder of LCA, which played a critical role in mediating these effects. Therefore, LCAbinding glycans may function as a novel indicator to predict metastasis for patients with EC.

\section{Supplementary Material}

Supplementary table S1.

http://www.jcancer.org/v11p4736s1.xls

\section{Acknowledgments}

This study was supported by financial grants from the National Natural Science Foundation of China (81902494), the Natural Science Foundation of Hubei Province (2019CFA034 and 2019CFB441), the Free Exploration Foundation of the Hubei University of Medicine (FDFR201802), and the Innovation Project for graduates of the Hubei University of Medicine (YC2019007).

\section{Competing Interests}

The authors have declared that no competing interest exists.

\section{References}

1. Bray F, Ferlay J, Soerjomataram I, et al. Global cancer statistics 2018: GLOBOCAN estimates of incidence and mortality worldwide for 36 cancers in 185 countries. CA Cancer J Clin. 2018; 68: 394-424.

2. Zeng H, Zheng R, Zhang S, et al. Esophageal cancer statistics in China, 2011: Estimates based on 177 cancer registries. Thorac Cancer. 2016; 7: 232-7.

3. Zhou Y, Chu L, Wang Q, et al. CD59 is a potential biomarker of esophageal squamous cell carcinoma radioresistance by affecting DNA repair. Cell Death Dis. 2018; 9: 887.

4. Hou W, Qiu Y, Hashimoto N, et al. A systematic framework to derive $\mathrm{N}$-glycan biosynthesis process and the automated construction of glycosylation networks. BMC Bioinformatics. 2016; 17 (Suppl 7): 240.

5. Stowell SR, Ju T, Cummings RD. Protein glycosylation in cancer. Annu Rev Pathol. 2015; 10: 473-510. 
6. Vasconcelos-Dos-Santos A, Oliveira IA, Lucena MC, et al. Biosynthetic machinery involved in aberrant glycosylation: promising targets for developing of drugs against cancer. Front Oncol. 2015; 5: 138.

7. Wang H, Yang X, Guo Y, et al. HERG1 promotes esophageal squamous cell carcinoma growth and metastasis through TXNDC5 by activating the PI3K/AKT pathway. J Exp Clin Cancer Res. 2019; 38: 324.

8. Lin MC, Huang MJ, Liu CH, et al. GALNT2 enhances migration and invasion of oral squamous cell carcinoma by regulating EGFR glycosylation and activity. Oral Oncol. 2014; 50: 478-84.

9. Suzuki O, Abe M, Hashimoto Y. Sialylation and glycosylation modulate cell adhesion and invasion to extracellular matrix in human malignant lymphoma: Dependency on integrin and the Rho GTPase family. Int J Oncol. 2015; 47: 2091-9.

10. Taniguchi N, Kizuka Y. Glycans and cancer: role of N-glycans in cancer biomarker, progression and metastasis, and therapeutics. Adv Cancer Res. 2015; 126: 11-51.

11. Pinho SS, Reis CA. Glycosylation in cancer: mechanisms and clinical implications. Nat Rev Cancer. 2015; 15: 540-55.

12. Cui J, Huang $\mathrm{W}, \mathrm{Wu} B$, et al. N-glycosylation by $\mathrm{N}$-acetylglucosaminyltransferase V enhances the interaction of CD147/basigin with integrin $\beta 1$ and promotes HCC metastasis. J Pathol. 2018; 245: 41-52.

13. Zhang C, Deng X, Qiu L, et al. Knockdown of C1GalT1 inhibits radioresistance of human esophageal cancer cells through modifying $\beta 1$-integrin glycosylation. J Cancer. 2018; 9: 2666-77.

14. Huang $\mathrm{WL}, \mathrm{Li} Y \mathrm{YG}, \mathrm{Lv}$ YC, et al. Use of lectin microarray to differentiate gastric cancer from gastric ulcer. World J Gastroenterol. 2014; 20: 5474-82.

15. Hirabayashi J, Kuno A, Tateno H. Development and applications of the lectin microarray. Top Curr Chem. 2015; 367: 105-24.

16. Jian $Q$, Yang Z, Shu J, et al. Lectin BS-I inhibits cell migration and invasion via AKT/GSK-3 $\beta / \beta$-catenin pathway in hepatocellular carcinoma. J Cell Mol Med. 2018; 22: 315-29.

17. Shen L, Luo Z, Wu J, et al. Enhanced expression of a2,3-linked sialic acids promotes gastric cancer cell metastasis and correlates with poor prognosis. Int Oncol. 2017; 50: 1201-10.

18. Zhou SM, Cheng L, Guo SJ, et al. Lectin RCA-I specifically binds to metastasis-associated cell surface glycans in triple-negative breast cancer. Breast Cancer Res. 2015; 17: 36.

19. Zhang X, Tian T, Liu C, et al. Elevated CD147 expression is associated with shorter overall survival in non-small cell lung cancer. Oncotarget. 2017; 8: 37673-80

20. Jimenez-Arellanes A, Luna-Herrera J, Ruiz-Nicolas R, et al. Antiprotozoal and antimycobacterial activities of Persea americana seeds. BMC Complement Altern Med. 2013; 13: 109.

21. Shen $\mathrm{L}$, Dong $\mathrm{X}, \mathrm{Yu} \mathrm{M}$, et al. $\beta 3 \mathrm{GnT}$ 8 promotes gastric cancer invasion by regulating the glycosylation of CD147. J Cancer. 2017; 8: 314-22.

22. Liu Y, Li L, Geng P, et al. RECK inhibits cervical cancer cell migration and invasion by promoting p53 signaling pathway. J Cell Biochem. 2018; 119: 3058-66.

23. Li BL, Lu W, Qu JJ, et al. Loss of exosomal miR-148b from cancer-associated fibroblasts promotes endometrial cancer cell invasion and cancer metastasis. J Cell Physiol. 2019; 234: 2943-53.

24. Cho HJ, Park JH, Nam JH, et al. Ascochlorin suppresses MMP-2-mediated migration and invasion by targeting FAK and JAK-STAT signaling cascades. J Cell Biochem. 2018; 119: 300-13.

25. Yin X, Liu Z, Zhu P, et al. CXCL12/CXCR4 promotes proliferation, migration, and invasion of adamantinomatous craniopharyngiomas via PI3K/AKT signal pathway. J Cell Biochem. 2019; 120: 9724-36.

26. Zhu JF, Huang W, Yi HM, et al. Annexin A1-suppressed autophagy promotes nasopharyngeal carcinoma cell invasion and metastasis by PI3K/AKT signaling activation. Cell Death Dis. 2018; 9: 1154

27. de Freitas Junior JC, Silva Bdu R, de Souza WF, et al. Inhibition of N-linked glycosylation by tunicamycin induces E-cadherin-mediated cell-cell adhesion and inhibits cell proliferation in undifferentiated human colon cancer cells. Cancer Chemother Pharmacol. 2011; 68: 227-38.

28. Khan I, Steeg PS. Metastasis suppressors: functional pathways. Lab Invest. 2018; 98: 198-210.

29. Subramanian SP, Babu P, Palakodeti D, et al. Identification of multiple isomeric core chitobiose-modified high-mannose and paucimannose $\mathrm{N}$-glycans in the planarian Schmidtea mediterranea. J Biol Chem. 2018; 293: 6707-20

30. Yang L, Nyalwidhe JO, Guo S, et al. Targeted identification of metastasis-associated cell-surface sialoglycoproteins in prostate cancer. Mol Cell Proteomics. 2011; 10: M110 007294.

31. Yang L, Song X, Zhu J, et al. Tumor suppressor microRNA-34a inhibits cell migration and invasion by targeting MMP-2/MMP-9/FNDC3B in esophageal squamous cell carcinoma. Int J Oncol. 2017; 51: 378-88.

32. Wang Y, Zang W, Du Y, et al. Mir-655 up-regulation suppresses cell invasion by targeting pituitary tumor-transforming gene-1 in esophageal squamous cell carcinoma. J Transl Med. 2013. 11: 301.

33. Wang L, Zhang Z, Yu X, et al. SOX9/miR-203a axis drives PI3K/AKT signaling to promote esophageal cancer progression. Cancer Lett. 2020; 468: $14-26$.

34. Liu C, Qiu H, Lin D, et al. c-Jun-dependent $\beta 3$ GnT8 promotes tumorigenesis and metastasis of hepatocellular carcinoma by inducing CD147 glycosylation and altering N-glycan patterns. Oncotarget. 2018; 9: 18327-40.
35. Fan J, Wang S, Yu S, et al. N-acetylglucosaminyltransferase IVa regulates metastatic potential of mouse hepatocarcinoma cells through glycosylation of CD147. Glycoconj J. 2012; 29: 323-34.

36. Chen J, Pan Y, He B, et al. Inhibition of CD147 expression by RNA interference reduces proliferation, invasion and increases chemosensitivity in cancer stem cell-like HT-29 cells. Int J Oncol. 2015; 47: 1476-84.

37. Hao J, Madigan $\mathrm{MC}$, Khatri $\mathrm{A}$, et al. In vitro and in vivo prostate cancer metastasis and chemoresistance can be modulated by expression of either CD44 or CD147. PLoS ONE. 2012; 7: e40716.

38. $\mathrm{Li} \mathrm{H}, \mathrm{Xu} \mathrm{Y}$. CD147 as a novel biomarker for predicting the prognosis and clinicopathological features of bladder cancer: a meta-analysis. Oncotarget. 2017; 8: 62573-88

39. $\mathrm{Li} \mathrm{H}$, Jiang $\mathrm{C}, \mathrm{Wu} \mathrm{D}$, et al. The prognostic and clinicopathologic characteristics of CD147 and esophagus cancer: A meta-analysis. PLoS ONE. 2017; 12: e0180271.

40. Zheng HC, Gong BC. CD147 expression was positively linked to aggressiveness and worse prognosis of gastric cancer: a meta and bioinformatics analysis. Oncotarget. 2017; 8: 90358-70. 